\title{
Management of dystonia in patients with hypersensitivity to anticholinergics
}

\author{
Ashish Sarangi MD, Lance Mwangi BA, E.L. Domingo-Johnson BSN, Arham Siddiqui BA
}

\begin{abstract}
Background: Anticholinergics are one of the first-line treatments for patients with dystonia. However, there are few practical guidelines for patients with dystonia who have developed hypersensitivity to anticholinergics.

Methods and Findings: This update was developed to provide clinicians with specific alternatives if patients are hypersensitive to anticholinergics but require management of dystonia. Recommendations were based upon literature review and expert opinion.

Conclusions: We provide the guidance on patients with dystonia who are hypersensitive to anticholinergics, based on a review of the literature. We propose personalized dystonia treatment approaches when anticholinergic hypersensitivity arises. Gaps in the existing literature and future directions in the treatment of anticholinergic hypersensitive patients being treated for dystonia are also highlighted.
\end{abstract}

Keywords: Dystonia, anticholinergics, hypersensitivity, antihistamine, cognitive behavior

\section{INTRODUCTION}

Although dystonia is a rare and uncommon disorder, it can be life threatening, especially when involving the laryngeal muscles causing laryngospasm. ${ }^{1}$ Patients with laryngospasm require emergency tracheotomy or intubation and intensive care.

Anticholinergics are the usual standard of care in management of dystonia, and in most cases, they are effective and safe. The treatment of dystonia has evolved, and more options are available in the management of this disorder. Despite this, there have been few reviews that adequately address the risks associated with the development of hypersensitivity reactions to antihistamines and how they should be managed. This paper compiles relevant PubMed articles over

Corresponding author: Ashish Sarangi Contact Information: Ashish.Sarangi@ttuhsc.edu DOI: 10.12746/swrccc.v9i39.765 the last 3 decades with an emphasis on the management of patients with multiple medications who have dystonia, on the treatment available for those who are allergic to anticholinergics, and on some of the relevant symptoms seen in those who develop hypersensitivities to anticholinergics.

Initially, dystonia was categorized into two groups following the First International Dystonia Symposium in 1975; the first was primary dystonia, which referred to how heritable the condition was (Huntington's, Wilsons, and neuroferritinopathy), and the second was dystonia due to environmental conditions (carbon monoxide, viral infection, and levodopa antagonists). Fahn (2013) later added "dystonia plus," referring to dystonia associated with another movement disorder. ${ }^{1} \mathrm{He}$ also expanded the classification system by the addition of autosomal dominant conditions to the existing categories of acquired and environmental causes of dystonia. $^{2}$

More recently, dystonia has been redefined as uncontrollable muscle contractions that can cause a person's body parts to twist involuntarily and result 
in repetitive movements or abnormal positions. The etiology of this condition is now categorized by two axes. The first axis includes clinical features, such as the age of onset, body distribution of the condition, temporal pattern (referring to whether or not it's progressive or static), duration following onset, and associated features. The second axis focuses on the etiology of the dystonia. It is classified as a sequela of nervous system pathology, a heritable condition, and, if all else is excluded, an idiopathic dystonia. ${ }^{3}$ Use of the etiology-based classification system allows for a more effective diagnosis and management of the causes of dystonia.

\section{THERAPEUTIC MANAGEMENT OF DYSTONIA}

Dystonia is typically managed by three main methods. The first is pharmaceutical management, then surgical management, and botulinum for the treatment of focal dystonia. The ABCDs of dystonia cover the most common medical treatment for dystonia; anticholinergics, baclofen, clonazepam, and dopamine-like medication. These classes target three main receptor systems, the GABA, cholinergic, and dopaminergic systems. ${ }^{4}$ Instead of anticholinergics, there are other treatment options available. Baclofen, a muscle relaxant, activates the GABA-B receptor and is most effective in treating craniofacial dystonias. Intra-thecal baclofen is also beneficial in the treatment of dystonia secondary to another medical condition, such as cerebral palsy. Anecdotal evidence suggests that some people may respond to baclofen for generalized dystonia, but its mechanism of action is poorly understood. ${ }^{5}$ Recommendations are to start with the lowest tolerable dose, usually $30 \mathrm{mg}$ spaced out in 3-4 doses, and to slowly down titrate the medication if side effects occur to avoid withdrawal.

\section{Potential drugs}

Several drugs are in clinical practice to treat or control the dystonia (Figure 1). The most common are:

Benzodiazepines are one option for those who need acute management of their dystonia to mainly treat

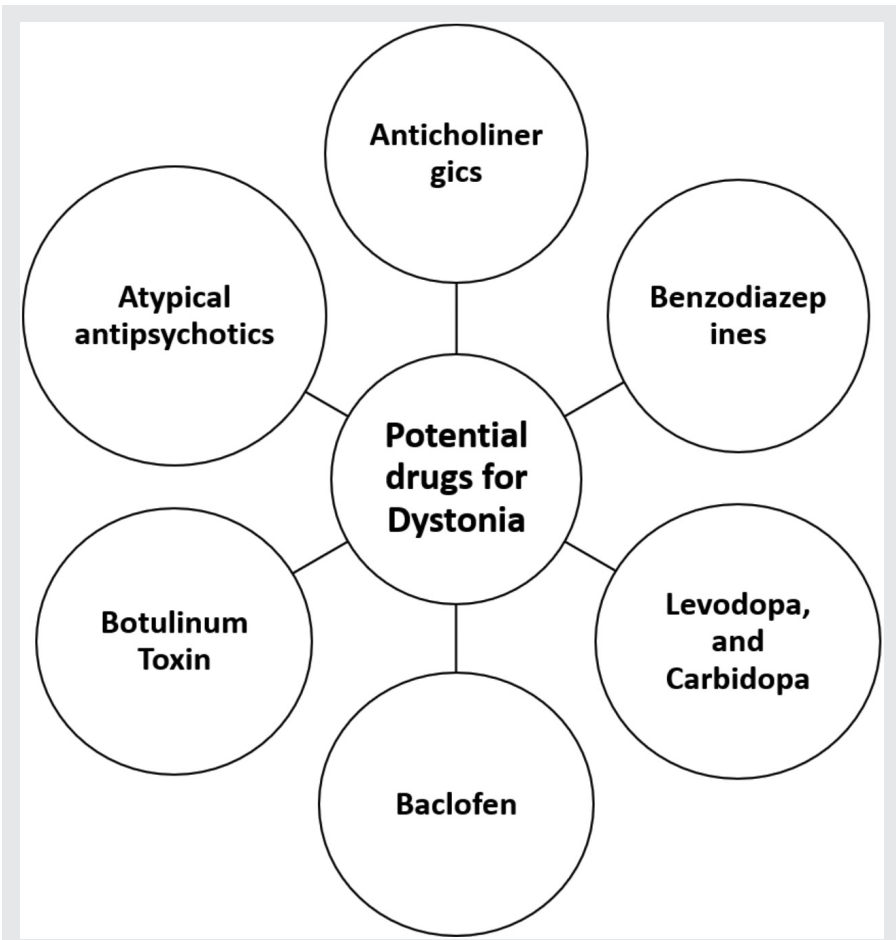

Figure 1. The most common drugs currently used to treat dystonia patients.

spasticity. Benzodiazepines are used sparingly due to their sedative and cardio-respiratory inhibiting effects that worsen with age. Very little information is known about the efficacy of these medications in treating dystonia, so there aren't any official guidelines. Recommendations are to start with clonazepam 0.5-10 mg QD, diazepam 2-100 mg QD, or lorazepam 0.5-8 mg QD. ${ }^{6}$

Levodopa and Carbidopa can be used to treat those who have early onset dystonia, such as a defect with tyrosine hydroxylase, or Segawa syndrome, which involves a defect in the synthesis of tetrahydrobiopterin. In those who have focal dystonia or dystonia that affects certain body parts, botulinum toxin can be used.

Baclofen is a presynaptic GABA agonist that is often used to treat spasticity but also has some effect on dystonia via an unknown mechanism. In a retrospective study, $20 \%$ of patients had a good response to baclofen in treating mild to moderate dystonia. They also found that there was significant improvement in children who 
were treated for primary idiopathic dystonia. Of the 31 children in the study, 30\% responded. The average dose used to treat them was $79 \mathrm{mg}$ per day. Some benefit has also been seen in children with early-onset primary dystonia in those with DYT1 mutations.

Atypical antipsychotics, such as quetiapine and risperidone, have been reported to be effective in treating primary dystonia. Clonazepam has also been found to have some effectiveness in treating axial, segmental, and generalized dystonia. The doses used for treatment were typically $12.5 \mathrm{mg}$ per day with $12.5-25 \mathrm{mg}$ incremental increases per week until symptomatic improvement is observed.

Botulinum toxin (BTX) is the treatment of choice for patients with focal or segmental dystonia, as well as blepharospasm, spasmodic dystonia, cervical, oromandibular, and lingual dystonia. ${ }^{7}$ While standard treatment with botulinum toxin has proven to be beneficial for the majority of those afflicted with cervical dystonia, there remains a small subset that is refractory to initial treatment. In that subset, it may be beneficial to try guided BTX administration via CT/PET. Recent reports suggest that treatment with BTX following imaging can help improve the responsiveness of those who initially appear unresponsive to the first treatment. ${ }^{8}$

\section{Anticholinergics}

At present, the most effective drugs against dystonia are anticholinergics. Figure 2 shows the basic structure of an anticholinergic.

\section{Anticholinergics AND DYSTONIA}

Several anticholinergics, such as trihexyphenidyl, benztropine, ethopropazine, procyclidine, and biperiden, have been used in the treatment of dystonia. Typically, anticholinergics act against the M1 receptor, but biperiden binds to nicotinic receptors, and procyclidine binds to $\mathrm{M} 2$ and $\mathrm{M} 4$ receptors. The most effective anticholinergic appears to be trihexyphenidyl. Burke found that patients with dystonia who were on a $30 \mathrm{mg}$ daily dose of trihexyphenidyl over 36 weeks had significant improvement in their symptoms in $71 \%$ of the 31 patients. $^{9}$

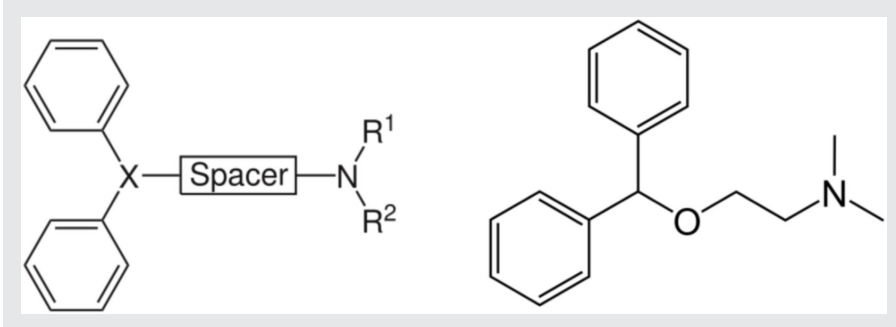

Figure 2. Structure of $\mathrm{H} 1$ antagonist (left) and diphenhydramine (right).

\section{HYPERSENSITIVITIES TO ANTIHISTAMINES}

The use of antihistamines for conditions and disorders, such as dystonia, does not anticipate concomitant hypersensitivity; however, the study of this risk and its associated effects allows further study of first-line treatment of these special populations who cannot tolerate anticholinergic agents. Instances of allergic reactions to antihistamines include the onset of urticaria from $\mathrm{H} 1$-antihistamines. A patient who presented with generalized urticaria was given a treatment of dexchlorpheniramine yet had increased symptoms. Follow up treatment with hydroxyzine did not remediate the urticaria either. Another patient seeking treatment for acute urticaria received mequitazine and ebastine, which caused erythematous lesions, angioedema, and generalized pruritus. ${ }^{10} \mathrm{Hydroxyzine}$ has also been reported to induce exanthematous pustulosis and anaphylaxis. ${ }^{11}$ Topical antihistamines, such as doxepin cream, can cause contact dermatitis. ${ }^{11}$ Cetirizine can rarely cause a delayed hypersensitivity reaction consistent with fixed-drug eruption. ${ }^{12}$ It is a challenge to define a causal relationship in these infrequent cases as symptoms can be similar to that of the primary disease being treated. Diagnoses are established using clinical suspicion and challenge testing. ${ }^{12}$

Recognizing the potential for hypersensitivity leads providers to prepare for the management of these reactions (Figure 3). Some patients who have had hypersensitivity reactions and without rescue medication have had physical symptoms (e.g., lesions) disappear over several hours. ${ }^{13}$ However, there are different approaches to the management of hypersensitivities depending on the situation. For example, an 


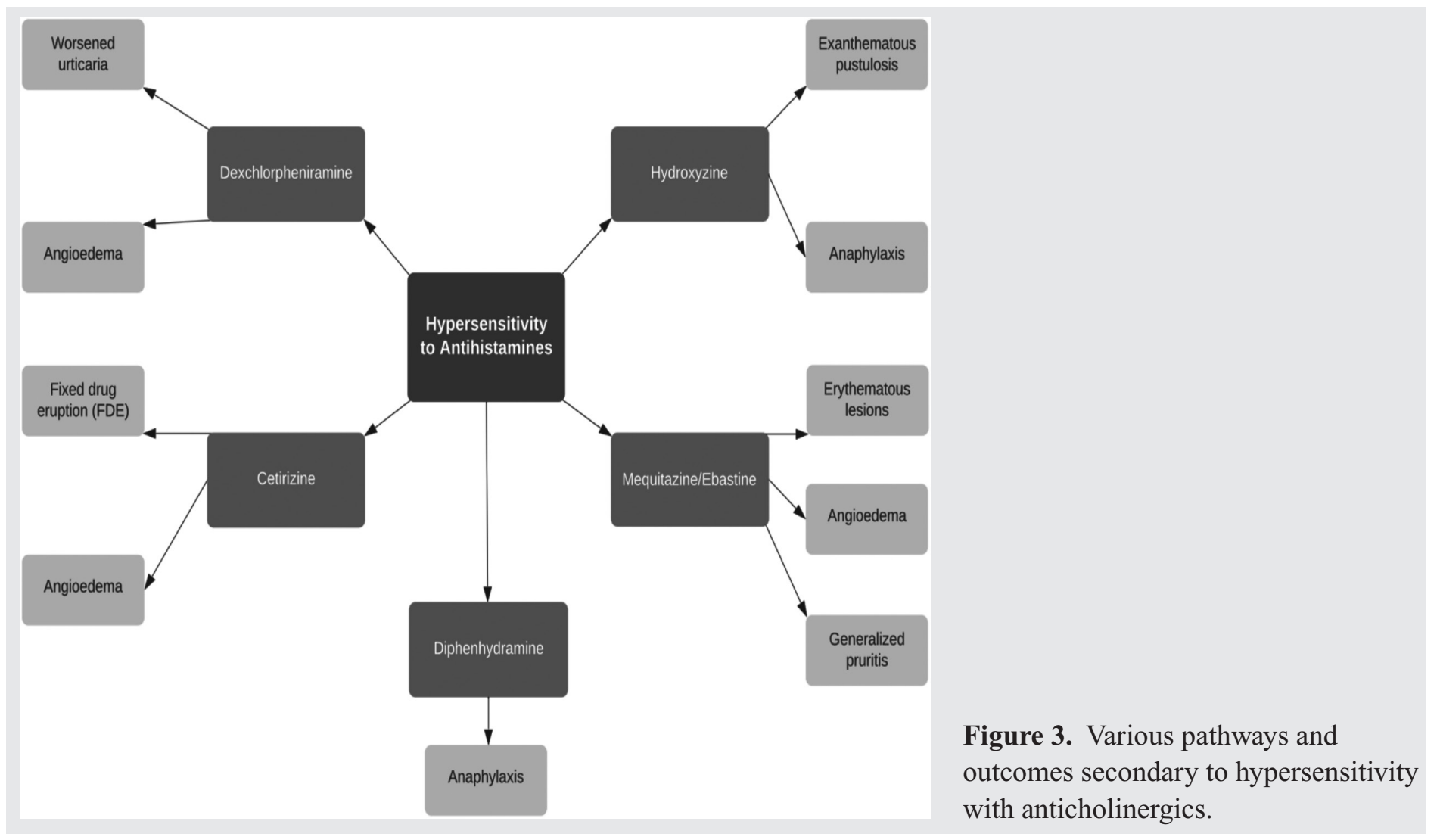

anaesthetized patient was given terbutaline, budesonide spray, and methylprednisolone to alleviate bronchospasm due to an anaphylactic reaction from previously administered hydroxyzine. ${ }^{14}$ Another bronchospasm episode forced surgery cancellation and continuous administration of terbutaline. This rare case of an allergic reaction to hydroxyzine raises the question whether antihistamine treatment in this patient could be used, and if so, which one. Cetirizine was found to be unsuitable but dexchlorpheniramine was tested and proposed as a premedication. This study highlights the importance of screening tests for antihistamines in special populations as a method of managing these hypersensitivities. ${ }^{14}$ In another case, a patient received multiple $\mathrm{H} 1$-antihistamines (cetirizine, ebastine, rupatadine, loratadine, and dexchlorpheniramine) for urticaria which triggered angioedema necessitating corticosteroid treatment. ${ }^{14}$ A specific protocol for the management of hypersensitivity with antihistamines has not been widely studied or established, and the rare reports have emphasized controlling the secondary allergic reaction symptoms (e.g., terbutaline for bronchospasm or corticosteroids for angioedema) and screening for other antihistamines that might trigger a similar response in the patient. The studies referenced suggest a unique portfolio of tolerable antihistamines for these special patient populations and management with alternate medication suitable to the patient. A limitation of this approach is evident from a report of a patient who developed intolerance to all the study's tested antihistamines. ${ }^{2,3}$ Studies have shown a positive prognosis for patients in alleviating secondary hypersensitivity responses. There is no superior treatment option as patient responses to antihistamines vary.

These findings are of interest in approaching anticholinergic treatment plans for those with dystonia. Diphenhydramine is classified as an anticholinergic and antihistaminic drug that has been shown to effectively treat idiopathic dystonia. ${ }^{5}$ However, several 
studies show the uncommon case of diphenhydramine induced anaphylaxis. Although hypersensitive to this agent, patients presented without cross-reactivity to other antihistamine drugs. Reported instances include diphenhydramine hypersensitivity with no cross-reactivity to loratadine ${ }^{6}$ terfenadine, cetirizine, loratadine, and dexchlorpheniramine ${ }^{9}$ and loratadine and cetirizine. $^{10,11}$ Although the patients had rare hypersensitivity to diphenhydramine, their tolerance to other antihistamines varies. This supports the idea that the best treatment for these special populations is unique to the patient. Nonetheless, conclusions can be drawn, including the fact that all three patients had no cross-reactivity to loratadine. A study of anticholinergic activity in $\mathrm{H} 1$ antihistamines showed that loratadine was closest to diphenhydramine in anticholinergic potency. Loratadine actually had higher anticholinergic activity than diphenhydramine. ${ }^{14}$ Although not a definitive treatment option, the presence of antihistamine with anticholinergics (and vice-versa) opens possibilities for managing dystonia in special populations with these hypersensitivities.

It is important to know about rare relationships between drugs and diseases/disorders. Diphenhydramine is an anticholinergic and antihistaminic agent which may have unintended hypersensitivities when used to treat allergies/dystonia; however, there is a case of a patient who suffered from diphenhydramine-induced dystonia. Alternate treatment for dystonia including diazepam and benztropine was given to alleviate the symptoms. Diphenhydramine may cause dystonia in one patient and treat the disorder in another; the main point is to make the clinicians aware of rare drug-induced conditions to ensure proper care of these patients.

\section{Conclusion}

This clinical review provides guidance for patients with dystonia who are hypersensitive to anticholinergics. Physicians should be diligent when ascertaining the reaction of patients who are hypersensitive to anticholinergics and adjust accordingly. This update can provide physicians with specific alternatives if this issue arises. More reports when patients are found to be hypersensitive to anticholinergics will help provide treatment guidelines and strategies.

Article citation: Sarangi A, Lance Mwangi L, DomingoJohnson EL, Siddiqui A. Management of dystonia in patients with hypersensitivity to anticholinergics. The Southwest Respiratory and Critical Care Chronicles 2021;9(39):35-40

From: Department of Psychiatry, Texas Tech University Health Sciences Center, Lubbock, Texas

Submitted: $10 / 3 / 2020$

Accepted: 4/3/2021

Reviewer: Kenneth Nugent MD

Conflicts of interest: none

This work is licensed under a Creative Commons Attribution-ShareAlike 4.0 International License.

\section{REFERENCES}

1. Albanese A, Bhatia K, Bressman SB, et al. Phenomenology and classification of dystonia: a consensus update. Mov Disord 2013;28:863-73.

2. Neychev VK, Gross RE, Lehéricy S, et al. The functional neuroanatomy of dystonia. Neurobiol Dis 2011;42:185-201.

3. Álvarez M, Quintanal N, Díaz A, et al. Dystonia and tremor secondary to thalamic infarction successfully treated with thalamotomy of the ventralis intermedius nucleus. Mov Disord 2014;29:1188-90.

4. Bragg DC, Sharma N. Update on treatments for dystonia. Curr Neurol Neurosci Rep 2014;14:454.

5. Termsarasab P, Thammongkolchai T, Frucht SJ. Medical treatment of dystonia. J Clin Mov Disord 2016;3:19.

6. Cloud LJ, Jinnah HA. Treatment strategies for dystonia. Expert Opin Pharmacother 2010;11:5-15.

7. Lee SA, Choi JY, Oh BM. The effect of computed tomographyguided botulinum toxin injection on cervical dystonia, confirmed by a 9-month follow-up using positron emission tomography/computed tomography. Am J Phys Med Rehabil 2020;99:e7-e10.

8. Barnes PJ. The role of anticholinergics in chronic obstructive pulmonary disease. Am J Med 2004; 117 Suppl 12A: 24S-32S.

9. Cetinel B, Onal B. Rationale for the use of anticholinergic agents in overactive bladder with regard to central nervous system and cardiovascular system side effects. Korean J Urol 2013;54:806-15. 
10. Carranza-del Rio J, Clegg NJ, et al. Use of trihexyphenidyl in children with cerebral palsy. Pediatr Neurol 2011;44: 202-6.

11. Balmain BN, Sabapathy S, Louis M, et al. Aging and thermoregulatory control: The clinical implications of exercising under heat stress in older individuals. Biomed Res Int 2018;2018:8306154.
12. Durnas C, Loi CM, Cusack BJ. Hepatic drug metabolism and aging. Clin Pharmacokinet 1990;19:359-89.

13. Weinstein JR, Anderson S. The aging kidney: physiological changes. Adv Chronic Kidney Dis 2010;17:302-7.

14. Fong TG, Tulebaev SR, Inouye SK. Delirium in elderly adults: diagnosis, prevention and treatment. Nat Rev Neurol 2009;5:210-20. 